\title{
Dielectric Embedded ESPAR (DE-ESPAR) Antenna Array for Wireless Communications
}

\author{
Junwei Lu, Member, IEEE, David Ireland, and Robert Schlub, Member, IEEE
}

\begin{abstract}
A novel dielectric embedded electronically steerable passive array radiator (ESPAR) (DE-ESPAR) antenna array for mobile wireless communication terminal is developed by finite element method based numerical modeling technique. The size reduction of the seven-element monopole antenna was accomplished by embedding the array in a cylindrical rod of FIK ceramic complex with a relative permittivity around $\varepsilon_{r}=4.5$. Dielectric embedded prototypes of a seven-element ESPAR antenna array were modeled and experimentally characterized. Experimental and numerical results are in good agreement. The optimized antenna produced a horizontal directivity of $5.1 \mathrm{dBi}$ and return loss of $19 \mathrm{~dB}$ at $2.48 \mathrm{GHz}$. An overall volume reduction of $80 \%$ and footprint reduction of $50 \%$ were achieved.
\end{abstract}

Index Terms-Adaptive antennas, dielectric embedded antenna array, parasitic radiator, smart antennas.

\section{INTRODUCTION}

I $\mathrm{N}$ MOBILE terminals the size of the antenna is important, as portability is a key requirement. The rapid growth of the wireless communications market has resulted in new technologies being investigated to improve performance and usage of the available spectrum in the most efficient way. Smart antennas with controllable directionality are one promising candidate as they allow higher re-use of channels and increased system performance. A host of electrically beam steerable adaptive antenna arrays have existed for some time. Typically, each element is excited with different phases of the information signal to form radiation beams and nulls. However, inherent complexity has restricted the use of conventional adaptive arrays in massively produced wireless communications products, like mobile computing terminals. To address this, electrically steerable or switchable parasitic antenna arrays have been introduced [1], [2]. Phased array antennas can also be considered, however they typically require an inter-element spacing of greater than $0.5 \lambda_{0}$. In electronically steerable passive array radiator (ESPAR) antenna arrays [3], [4], the separation distances between elements can be reduced to $0.25 \lambda_{0}$.

The ESPAR antenna can direct radiation at intended recipients and steer radiation nulls toward the interfering signals. The radiation nulls and main lobe gains complement each other to maximize the system signal to interference noise ratio

Manuscript received December 16, 2004; revised March 25, 2005.

J. Lu and D. Ireland are with the School of Microelectronic Engineering, Griffith University, Brisbane QLD 411, Australia (e-mail: j.lu @ griffith.edu.au; d.island@griffith.edu.au).

R. Schlub is with Antenova Ltd., Cambridge CB5 8RN, U.K. (e-mail: Robert.Schlub@antenova.com).

Digital Object Identifier 10.1109/TAP.2005.852517

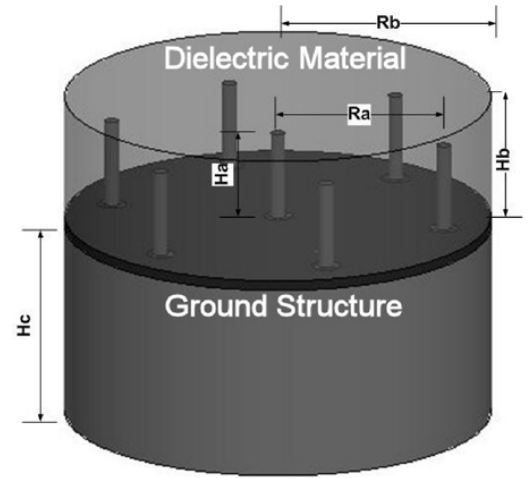

(a) Configuration of DE-ESPAR antenna array

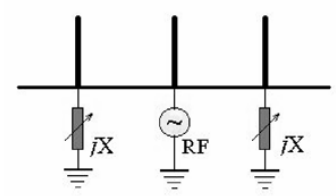

(b) Beamforming control circuits

Fig. 1. DE-ESPAR antenna structure.

(SINR). Furthermore, the antenna's main lobe gain dramatically decreases the required transmission power for a set range. Directed radiation also helps alleviate health concerns that might accompany such a device.

To further reduce the ESPAR antenna size, the DE-ESPAR antenna is developed and the physical size can be significantly reduced. Like ESPAR antenna the DE-ESPAR antenna consists of a single feed element surrounded by a ring of reactively loaded parasitic elements and is embedded into a high dielectric constant material as shown in Fig. 1(a). By electrically controlling the loading reactances [Fig. 1(b)], directional beams and nulls can be formed and steered throughout the azimuth. Thus the physical structure of the antenna is simple and requires relatively little power to operate when compared to its phased array counterparts. However, the strong electromagnetic coupling between elements in the dielectric material, and the interface matching between air and high dielectric materials, and finite ground structure make the antenna's analytical solution very complex. Accordingly, computational modeling is required to determine the antenna characteristics without experimental testing. Due to this complexity, much effort is needed to determine the optimum structure of the antenna for best performance.

In this paper, the work extends our earlier research work on ESPAR and DE-ESMB antenna arrays for mobile communications [4]-[6]. The size reduction was achieved by embedding the ESPAR antenna array in a homogeneous dielectric material. 
Small antenna design processes and analysis concepts are developed to analyze the problem based on computer modeling and optimization techniques. In order to optimize the DE-ESPAR antenna structure, a genetic algorithm (GA) was employed in conjunction with an finite element method (FEM) based cost function. The genetic optimization focused on improving the main-lobe horizontal gain of the antenna, and DE-ESPAR antenna structure. In doing so, both radiation sensitivity at side angles and transmitter power are reduced. In the majority of signal environments, it is conceivable that the transmitting and receiving antennas will be operating with positive gain in their propagation direction.

\section{CONFIGURATION OF DE-ESPAR ANTENNA AND LOAD}

Unlike conventional adaptive arrays, the DE-ESPAR antenna's beam forming elements are all passive in nature and do not require complex feeding networks and control systems. Typically, the parasitic elements are base-loaded with varactor diodes whose voltage controlled reactances produce different antenna radiation patterns [3], [4].

Similar to conventional adaptive arrays, the DE-ESPAR antenna is an adaptive solution that can create and steer both radiation lobes and nulls in arbitrary directions. A sevenelement DE-ESPAR antenna is considered where a single feed monopole is encircled by six, equally spaced, parasitic monopole elements are embedded by high dielectric constant material and reside on a hollowed cylindrical ground structure, under which the parasitic elements are loaded. While this ground configuration consumes substantially more volume than would be required for the complementary dipole array, it provides a practical, mechanically sound solution for antenna feeding and control circuitry. The ground skirting is used to reduce the radiation lobe elevation that exists due to the finite ground plane dimensions. Reducing the main-lobe elevation not only increases the azimuth gain and improve the communication performance of the wireless terminal devices, but also reduces the chances of multi-path interference.

DC voltage controlled varactors are the common component for implementing dynamic reactive loads. However for simulation verification, static loads consisting of a segment of microstrip transmission line (TL) were used for prototype testing. As the monopole elements were held in position by a FR4 Fiberglass printed circuit board (PCB), the microstrip lines were placed on the bottom side of the PCB while the topside obviously being the ground plane. The required lengths of the transmission lines were derived by a combination of analytical transmission line equations and manual simulation optimization. The Beamforming control circuit with combined load configuration using microstrip TL is shown in Fig. 2. Since varactors based load is a capacitive load, the maximum antenna efficiency (the function of structure detention and antenna gain) observed was only $79 \%$ in contrast to inductive based load, where $98 \%$ was observed. It can be seen that the antenna efficiency is influenced by load configuration. Therefore the DE-ESPAR antenna designed with inductive loads (Fig. 3) can significantly improve the antenna efficiency.

\section{Antenna Element}

\section{Transmission Line}

Microstrip

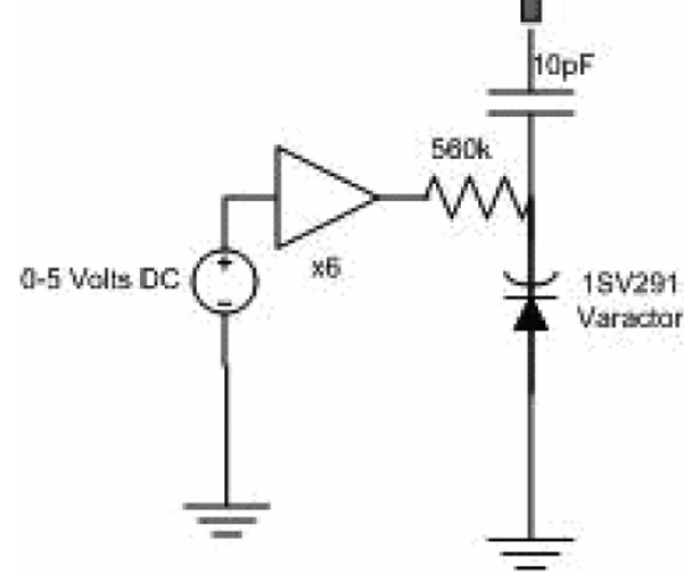

Fig. 2. Beamforming control circuit with combined load configuration.

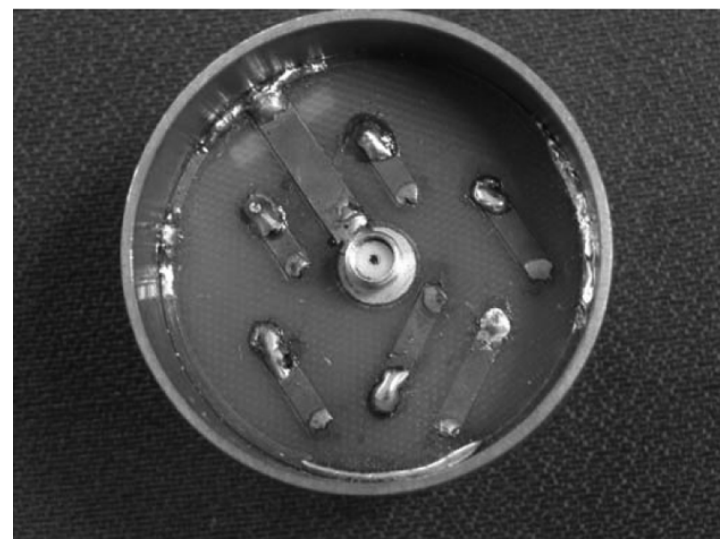

Fig. 3. Antenna static loads structure (for a fix beam direction).

Although the inductive load can provide many benefits, such as wide bandwidth of frequency and increased gain, the high frequency and low loss variable inductors cannot be easily found. Therefore, the combination of a quarter wave microstrip line in series with varactor is utilized for beam forming control circuit as shown in Fig. 4. With such configuration, the impedance changes at $2.4 \mathrm{GHz}$ from $\mathrm{j} 17 \Omega$ to $\mathrm{j} 170 \Omega$, in contrast to capacitive load the impedance changes from $-\mathrm{j} 14.73 \Omega$ to $-\mathrm{j} 147 \Omega$. 


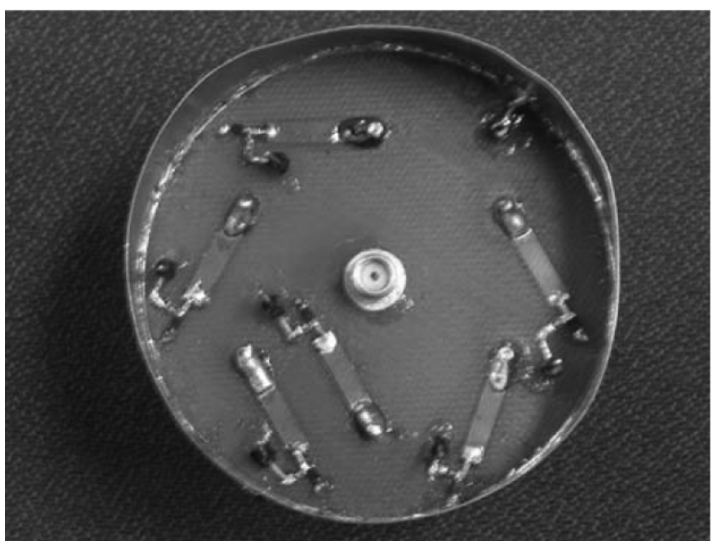

Fig. 4. Dynamically controlled load (varactor combined with equal length of microstrip TL).

Due to combined $1 / 4$ wave length transmission line, the DC controlled varactors allow simple electrically formed radiation with minimal power expense (Fig. 4). Its uncomplicated structure and low-power operation make the antenna an ideal candidate for massively produced user terminals in wireless communications network.

\section{OPtimization PROCEDURE IN Size REDUCTION}

It is well known that by embedding a wire in a dielectric medium, the electrical length of a wire antenna can be reduced. If the antenna elements are embedded in a high permittivity medium (nonmagnetic material) of infinite extent, the wavelength in the medium can be rewritten as $\lambda_{r}=\lambda_{0} / \sqrt{\varepsilon_{r}}$ and the size reduction factor $F_{r}$ for the length of antenna elements will be $F_{r}=\sqrt{\varepsilon_{r}}$.

To reduce the total volume and weight of the DE-ESPAR array, the radius of the dielectric from outer element to air interface should be smaller than the height of the dielectric. In a practical antenna design, the reduction factor will not be as great as $F_{r}$ due to the embedding dielectric will not be of infinite extent. Clearly, the reduction factor will be a function of the shape, size, electrical characteristics of the dielectric material and the location of each wire element in the material. Thus the actual reduction factor $F_{r a}$, for the size of antenna will be

$$
F_{r a}=f\left(\sqrt{\varepsilon_{r}}, R_{a}, R_{b}, H_{q}, H_{b}, H_{c}\right)
$$

where the parameters $R_{a}, R_{b} H_{a}, H_{b}$, and $H_{c}$ are defined in Fig. 1(a).

This inherent nature makes the formulation of an analytical solution quite arduous. Therefore the design methodology employed featured a FEM based simulating engine in conjunction with an automated GA as the optimization tool. The choice of the GA as an optimization technique was based around its recent success in optimizing similar antennas [4] and its resilience to being trapped in local minima. The FEM solution engine was used due to the complex structural nature of the DE-ESPAR antenna where a nonuniform mesh was implemented allowing efficient but accurate simulations.
TABLE I

OPTIMIZED LOAD VALUES $(j \Omega)$

\begin{tabular}{l|l|l|l|l|l}
\hline$x_{1}$ & $x_{2}$ & $x_{3}$ & $x_{4}$ & $x_{5}$ & $x_{6}$ \\
\hline 46 & 22 & -16 & -25 & -16 & 22 \\
\hline
\end{tabular}

TABLE II

OPTIMIZED ESPAR ANTENNA DIMENSIONS

\begin{tabular}{c|c|c|c|c|c}
\hline Length & $H_{a}$ & $H_{b}$ & $H_{c}$ & $R_{a}$ & $R_{b}$ \\
\hline$(\mathrm{mm})$ & 15.5 & 20 & 31 & 24 & 30 \\
\hline$\lambda$ & 0.124 & 0.16 & 0.248 & 0.192 & 0.24 \\
\hline
\end{tabular}

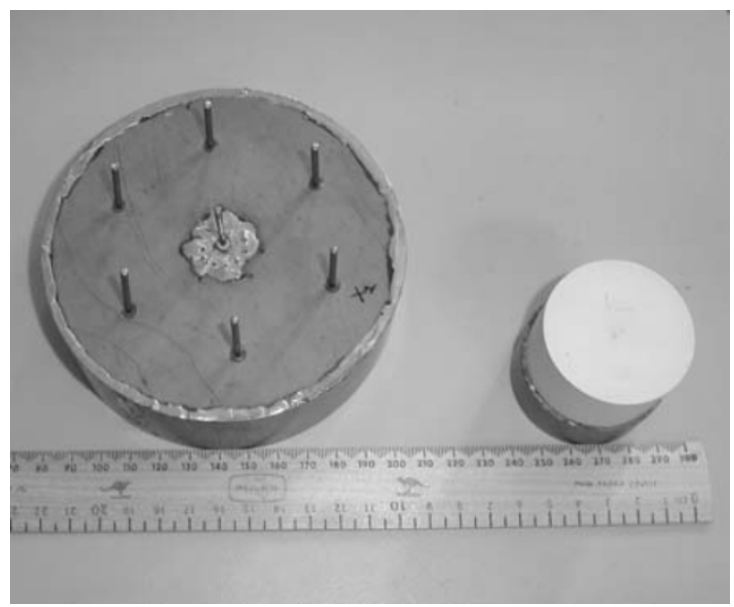

Fig. 5. Comparison of constructed free space ESPAR and DE-ESPAR antenna arrays.

\section{A. Structural Optimization}

The original structure (Fig. 1) was designed using size reduction factor $F_{r}$ and fundamental design techniques in conjunction with heuristic experience and therefore, it was not known whether all dimensions were optimal. While the structure is relatively simple, the electrical analysis of the antenna is complex, and hard to optimize analytically. Therefore, the finite element based commercial software package High Frequency Structure Simulator (HFSS) [7] was used together with a GA to simulate and optimize the structure.

Optimal design of the DE-ESPAR antenna includes two components, namely the structural and load optimization. Both components couple together to formulate the complete electromagnetic characteristics of the DE-ESPAR antenna array. Structural optimization involves the element heights, element array radius as well as the dielectric radius and height. To reduce optimization time, element structural variables were kept within limits that were determined by available material and the overall size of the antenna. The monopole elements were kept at $1 \mathrm{~mm}$ radius while the ground skirt was assumed a two-dimensional shape (0 mm thickness) in the simulation process as this parameter was assumed not to significantly influence the behavior of the antenna [8].

\section{B. Reactive Load Optimization}

DC voltage controlled varactor diodes are the typical component for implementing a dynamic reactive load. However, for 

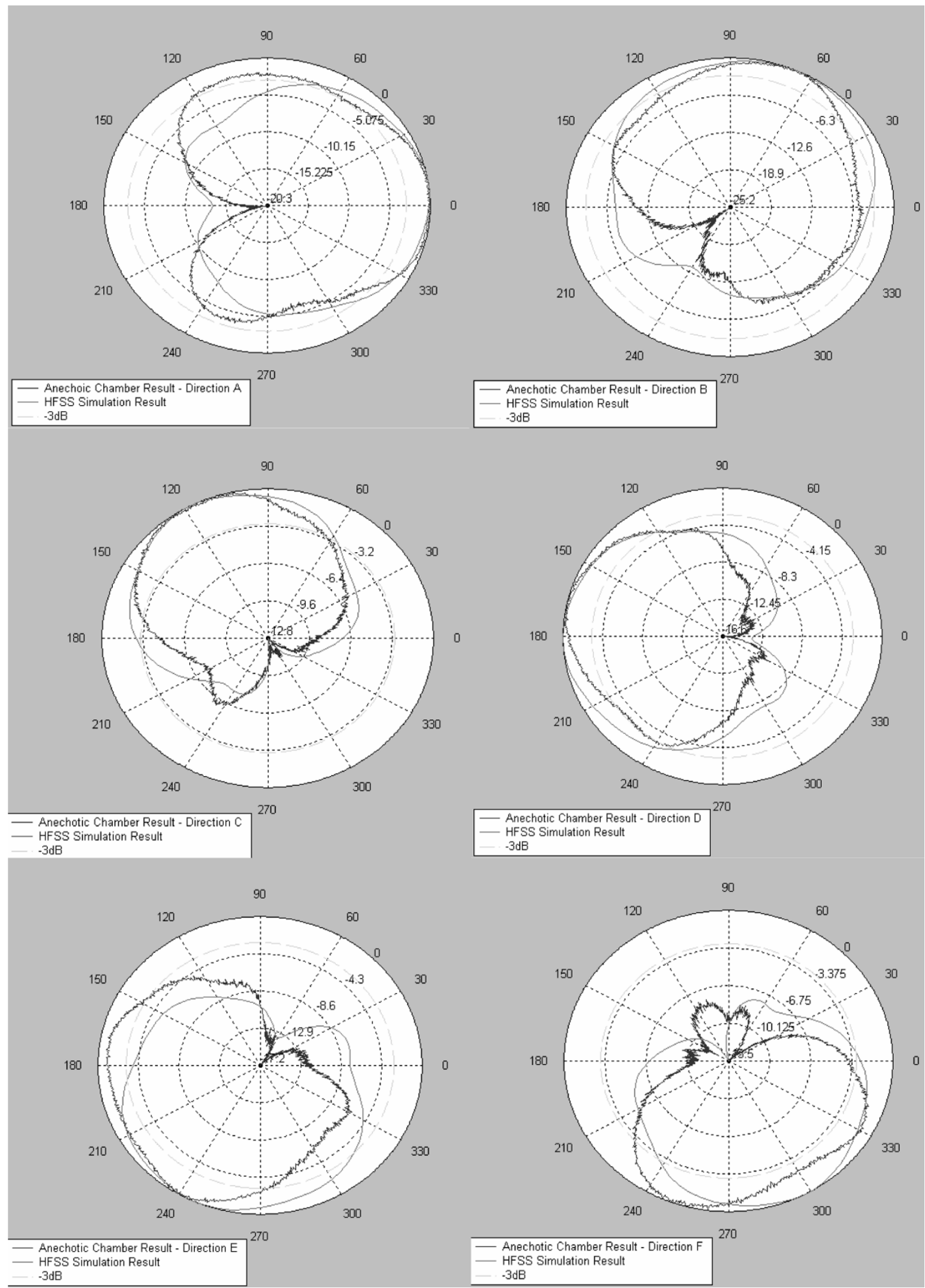

Fig. 6. Radiation beam in 6 directions (at $60^{\circ}$ intervals for each step) with inductive load and compared with experiment results, where the red thin line is experimental results and the blue rough line is simulated results.

simulation verification it is more convenient to realize static loads consisting of a segment of open or short circuited mi- crostrip transmission line (TL). Elementary transmission line theory states the input impedance of a lossless transmission line 
is obtained by the characteristic impedance $R_{o}$, the terminating impedance $Z_{L}$, the phase constant $\beta$ and the length of the transmission line $\ell$.

In the case of an open circuited transmission line $Z_{L}=\infty$ the resultant input impedance is given as, $Z_{\text {in }}=-j R_{0} / \tan (\beta \ell)$

In contrast the corresponding short circuit transmission line is derived by substituting $Z_{L}=0$ resulting in $Z_{\text {in }}=j R_{0} \tan (\beta \ell)$

By selecting either a short or open circuit transmission line and substituting in $R_{o}, Z_{\text {in }}$ and $\beta$ the required length of the transmission line will provide the desired load impedance during the optimization process.

\section{The GA}

It was anticipated that the optimization process would result in a large amount of suboptimal solutions calling upon a robust and global optimizer algorithm. The GA was the obvious choice as it exhibits such trade characteristics and also has recently proven successful in optimizing similar antenna structures [9]-[11].

A conventional GA was applied with the exception of an adaptive mutation parameter. Typically the mutation parameter in a GA is the amount of the random altering of the evolved population of solutions, promoting a nonhomogenous population. It is often the case when this parameter is fixed usually at a small amount (e.g., 1\%) the dispersion of the evolving population will decrease per iteration resulting in inefficient optimization. In contrast if the mutation rate is higher the optimization search then becomes random and the genetic method is loss. A compromise can be reached by adaptively varying the mutation value based upon the statistical dispersion of the evolved population $P_{d}$, normalized with the dispersion of the initial randomly created population $P_{o}$. The mutation parameter or rate $P_{m}$ is updated according to (2),

$$
P_{m}=\left(1-\frac{P_{d}^{2}}{P_{o}}\right) \times 100 \% .
$$

The two defining parameters of an antenna are its efficiency $\left(1-\left|S_{11}\right|^{2}\right)$ comprised of the reflection coefficient $\left(S_{11}\right)$ and directivity (D), assuming negligible losses in the antenna structure, the product of these two components formulates the overall gain of the antenna [4]. In the case of a wireless or mobile network it is conceivable to postulate an increase in horizontal gain will promote the most direct route of radiation energy to the intended receiver. With this in mind the implemented fitness function shown in (3), consisted simply of the horizontal gain with the objective of the optimizer to maximize this value.

$$
\text { FitnessFunction }=\left(1-\left|S_{11}\right|^{2}\right) D .
$$

Over 3000 simulations were performed by the optimizer resulting in 60 generations of evolved populations of solutions. The total optimization time was approximately one week with the use of two parallel processors. The optimized results for the reactive loads and structure dimensions at $2.4 \mathrm{GHz}$ are given in Tables I and II respectively. The optimized antenna structure revealed a significant size reduction in the element heights $\boldsymbol{H}_{\boldsymbol{a}}$ and radius of the element array $\boldsymbol{R}_{\boldsymbol{a}}$ with lengths of $0.124 \lambda$ and $0.192 \lambda$ respectively. The dielectric material height $\boldsymbol{H}_{\boldsymbol{b}}$ and radius $\boldsymbol{R}_{\boldsymbol{b}}$ were also found to be $0.16 \lambda$ and $0.24 \lambda$ respectively. The

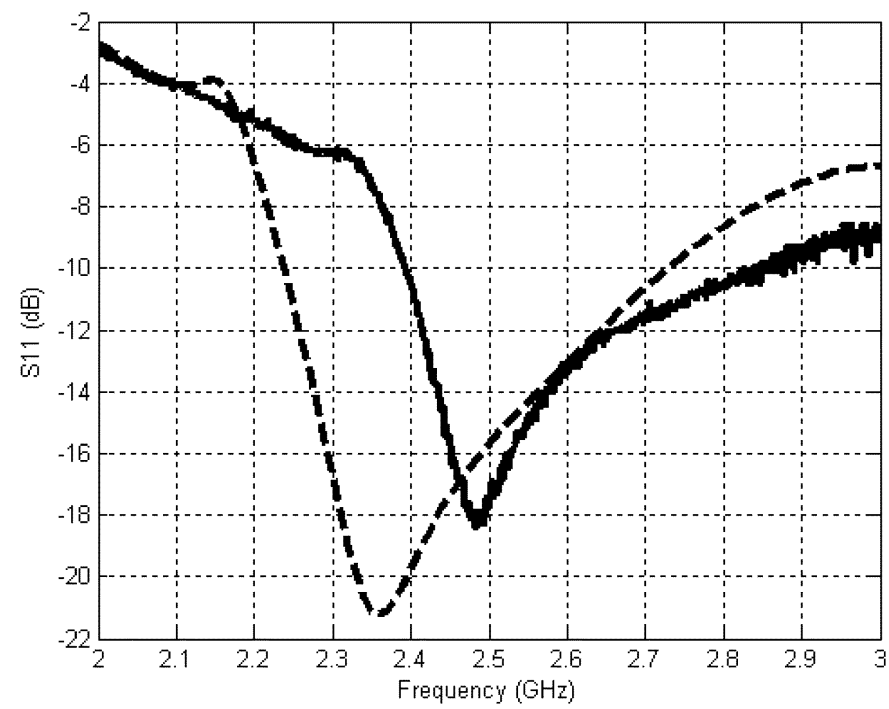

Fig. 7. DE-ESPAR reflection coefficient $\mathrm{S}_{11}$, where - - - - line indicates the simulation result of DE-ESPAR, and — line indicates experiment result of DE-ESPAR antenna.

cylindrical ground structure $\boldsymbol{H}_{\boldsymbol{c}}$ was found to have the largest dimension of $0.248 \lambda$. The measured $S_{11}$ at $2.4 \mathrm{GHz}$ revealed a value of $-11.5 \mathrm{~dB}$ while the optimized antenna exhibited a resonant frequency of approximately $2.48 \mathrm{GHz}$.

Fig. 5 show the constructed DE-ESPAR antenna (right side) compared with free space ESPAR antenna (left side). Obviously, the goal of size reduction has been significantly achieved. An overall volume reduction of $80 \%$ and footprint reduction of $50 \%$ were obtained. Like free space ESPAR antenna, DE-ESPAR antenna structure can be also simply made by hand within a short time. Dual plated PCB formed the antenna ground base on the topside and microstrip TL based inductive load mounted on the other side of the PCB while flexible copper sheeting made the skirt and the radiating elements were covered by dielectric block.

\section{OPTIMIZED DE-ESPAR ANTENNA RESULTS}

Fig. 6 shows the radiation pattern produced at $60^{\circ}$ intervals through the azimuth due to the antenna's symmetry. Since this prototype DE-ESPAR antenna was the hand-made product which did not meet the industry standard, therefore, the error between measured and simulated results for each radiation pattern can be clearly seen. Such manufacture errors do not effect the antenna performance and they are acceptable for conceptual prototype development.

Fig. 7 shows the statically loaded DE-ESPAR reflection coefficient $\mathrm{S}_{11}$. The measured $\mathrm{S}_{11}$ at $2.4 \mathrm{GHz}$ revealed a value of $-11.5 \mathrm{~dB}$ while the optimized antenna exhibited a resonant frequency of approximately $2.48 \mathrm{GHz}$ and a $10 \mathrm{~dB}$ bandwidth of approximately $450 \mathrm{MHz}$. It was found that the use of a double-sided PCB substrate and microstrip transmission line based components as the fixed impedances in the experimental model resulted in the shift in resonant frequency to $2.48 \mathrm{GHz}$. The simulated reflection coefficient compared with the measured result demonstrated some difference due to several reasons, namely the simulation model assumed the value of the 


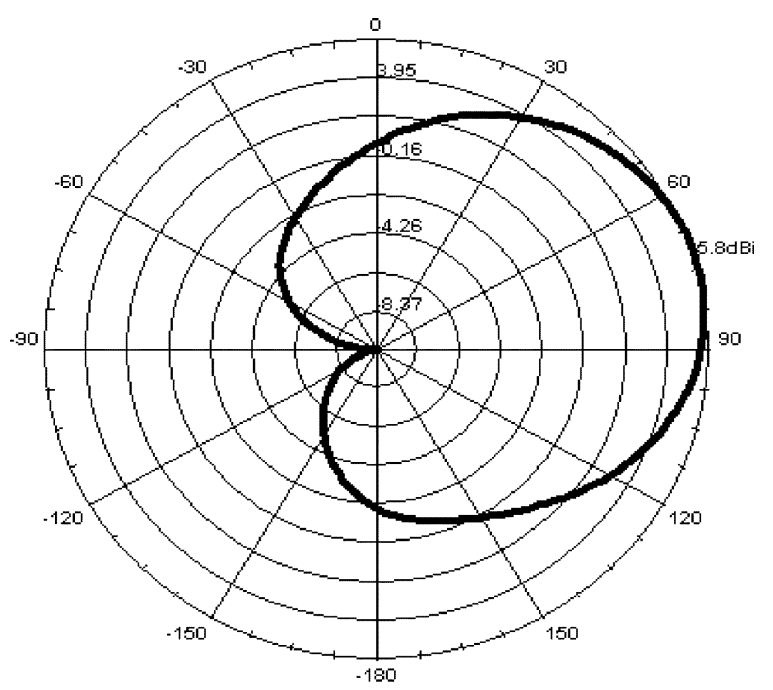

(a)

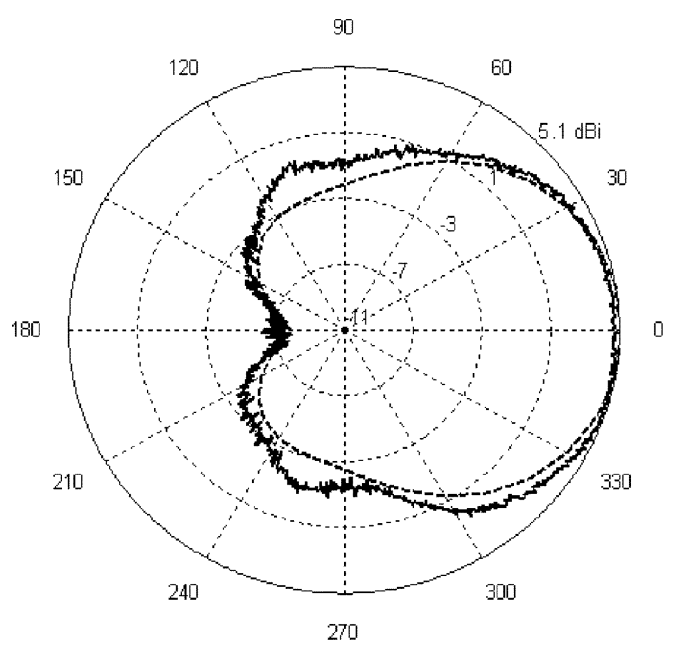

(b)

Fig. 8. E plane radiation $\left(\phi=90^{\circ}\right), \mathrm{H}$ plane radiation $\left(\theta=0^{\circ}\right)$ Radiation characteristics of the optimized DE-ESPAR antenna at $2.4 \mathrm{GHz}$; (a) simulated E plane radiation $\left(\phi=90^{\circ}\right)$, (b) $\mathrm{H}$ plane radiation $\left(\theta=0^{\circ}\right)$ where - measured, - - simulated.

impedance loads were constant over the frequency sweep where in fact they are quite dependent on frequency. Also the dielectric constant of the materials may not of been accurately defined in the simulation model as their values tend to vary.

The resultant radiation patterns of the statically loaded antenna at $2.4 \mathrm{GHz}$ can be seen in revealing a directivity of $5.1 \mathrm{dBi}$ and a beamwidth of $80^{\circ}$. Fig. 8 also shows a good agreement between the simulated and experimental results. Due to the symmetrical structure of the parasitic loaded elements the produced radiation pattern of the antenna can be rotated at $60^{\circ}$ intervals through the azimuth plane by interchanging the load values.

\section{CONCLUSION}

A DE-ESPAR antenna was presented with microstrip transmission line loads producing a maximum gain of $5.1 \mathrm{dBi}$. The embedded dielectric material with a $\varepsilon_{r}$ of 4.5 was found to effectively reduce the size of the monopole elements and array radius. An overall volume reduction of $80 \%$ and footprint reduction of $50 \%$ were achieved. Size reduction and the simplistic control system of this antenna make it a suitable candidate for commercialization in mobile wireless communications system.

The GA employed a FEM based cost function in order to obtain accurate modeling of the antenna. In this work both physical antenna structure and loading reactances were optimized. As a result, the optimized antenna structure revealed a significant size reduction in the element heights $\boldsymbol{H}_{\boldsymbol{a}}$ and radius of the element array $\boldsymbol{R}_{\boldsymbol{a}}$ with lengths of $0.124 \lambda$ and $0.192 \lambda$ respectively. The dielectric material height $\boldsymbol{H}_{\boldsymbol{b}}$ and radius $\boldsymbol{R}_{\boldsymbol{b}}$ were also found to be $0.16 \lambda$ and $0.24 \lambda$ respectively. The cylindrical ground structure $\boldsymbol{H}_{\boldsymbol{c}}$ was found to have the largest dimension of $0.248 \lambda$.

\section{REFERENCES}

[1] S. Preston, D. Thiel, and J. Lu, "A multibeam antenna using switched parasitic and switched active elements for space-division multiple access applications," IEICE Trans. Jpn., vol. E82, no. 7, pp. 1202-1210, 1999.

[2] D. V. Thiel and S. L. Smith, Switched Parasitic Antennas for Cellular Communications. Norwood, MA: Artech House, 2002.

[3] T. Ohira and K. Gyoda, "Electronically steerable passive array radiator antennas for low-cost analog adaptive beamforming," in Proc. IEEE Int. Conf. Phased Array Systems Technology, May 2000, pp. 101-104.

[4] R. Schlub, J. Lu, and T. Ohira, "Seven element ground skirt monopole ESPAR antenna design using a genetic algorithm and the finite element method," IEEE Trans. Antennas Propag., vol. 51, no. 11, pp. 3033-3039, Nov. 2003.

[5] J. Lu, D. Thiel, B. Hanna, and S. Saario, "Dielectric embedded electronically switched and multiple beam antenna arrays for wireless communications systems," Electron. Lett., vol. 37, no. 14, pp. 871-872, Jul. 2001.

[6] J. Lu, D. Thiel, and S. Saario, "FD-TD analysis of dielectric embedded electronically switched multiple-beam antenna array," IEEE Trans. Magn., vol. 38, no. 2, pp. 701-704, Mar. 2002.

[7] (2004) HFSS v9.0. Ansoft Corp.. [Online]. Available: http://www.ansoft.com

[8] Y. Ojiro, H. Kawakami, K. Gyoda, and T. Ohira, "Improvement of elevation directivity for ESPAR antennas with finite ground plane," in Proc. IEEE Antennas Propagat. Soc. Symp., 2001, pp. 18-19.

[9] E. E. Altshuler and D. S. Linden, "Design of a loaded monopole having hemisphericdal coverage using a genetic algorithm," IEEE Trans. Antennas Propag., vol. 45, pp. 1-4, Jan. 1997.

[10] Z. Altman, R. Mittra, and A. Boag, "New designs of ultra wide-band communication antennas using a genetic algorithm," IEEE Trans. Antennas Propag., vol. 45, no. 10, pp. 1494-1505, Oct. 1997.

[11] D. S. Weile and E. Michielssen, "Genetic algorithm optimization applied to electromagnetics: A review," IEEE Trans. Antennas Propag., vol. 45, no. 3, pp. 343-353, Mar. 1997.

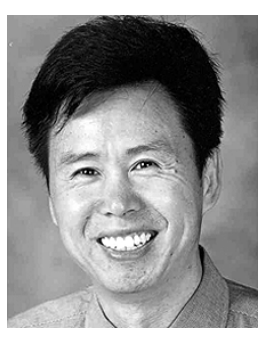

Junwei Lu (M'92) received the degree in electrical engineering from Xian Jiaotong University, China, in 1976, the M.Eng. degree in electronic and computer engineering from the National Toyama University, Toyama, Japan, in 1988, and the Ph.D. degree in electrical and computer engineering from the National Kanazawa University, Kanazawa, Japan, in 1991.

From 1976 to 1984, he worked with the Institute of Qinhai Electric Power Testing and Research, China, where he was involved in the various national research projects for electrical power industry. In 1985, his academic study and research was in the area of computational electromagnetics at the laboratory of electrical communications at Toyama University, Japan. In 1988, he worked on the applied computational electromagnetics and was involved in the development of magnetics devices with the Laboratory of Electrical Energy Conversion, Kanazawa University. He joined the School of Microelectronic Engineering, Griffith University, Brisbane, Australia, in 1992, where he is now an Associate Professor. His field of interest are computational and visual electromagnetics, high performance cluster computing, smart mobile terminal antennas and RF/MW devices and circuits, and high-frequency magnetics. 


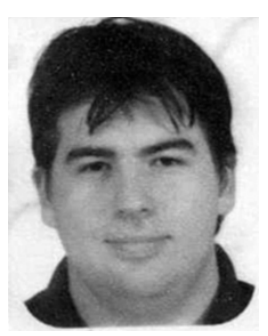

David Ireland received the B.Eng. degree in microelectronic engineering from the Griffith University, Brisbane, Australia, in 2004, where he is currently working toward the Masters of Philosophy.

His research interests include adaptive antennas, stochastic optimization techniques and numerical modeling techniques in electromagnetics.

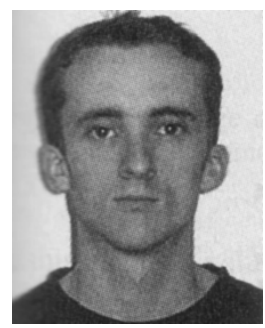

Robert Schlub (M'03) received the B.Eng. degree in microelectronic engineering and the Ph.D. degree from Griffith University, Brisbane, Australia, in 2000 and 2004, respectively.

$\mathrm{He}$ is currently designing handset antennas at Antenova Ltd., Cambridge, U.K. 